\title{
Fichte et la première philosophie de la nature de Schelling
}

\author{
Claude Piché, Université de Montréal
}

Ceci est la version post-publication (« postprint ») d'un article qui est paru sous forme abrégée dans : Dialogue, 43, 2004, p. 211-237.

RÉSUMÉ : Lorsque l'on reconstruit la philosophie fichtéenne de la nature de la période d'Iéna, on note des similitudes frappantes entre la conception de l'organisme telle que présentée dans la Doctrine de la Science et les développements correspondants de la première Naturphilosophie de Schelling. Or, bien que les deux penseurs s'entendent pour envisager la nature organique dans le cadre de l'idéalisme transcendantal, il est néanmoins possible à cette étape de déceler de légères divergences dans leur interprétation qui laissent présager leur futur différend sur le statut de la philosophie de la nature. Si, en l'occurrence, ils conviennent de considérer l'organisme comme un « analogon » de l'absolu, tout dépend alors de la manière dont chacun d'eux conçoit cette analogie : d'un point de vue pratique ou d'un point de vue théorique.

MOTS-CLÉS : Fichte, Schelling, Naturphilosophie, organisme, idéalisme transcendantal

ABSTRACT: When we reconstruct Fichte's philosophy of nature of the Jena period, we can notice striking similarities between the conception of organism in the Doctrine of Science and Schelling's corresponding developments in his early Naturphilosophie. But even though both thinkers agree to consider organic nature within the framework of transcendental idealism, it is nevertheless possible at this stage to discover slight differences in their interpretation, which announce their future disagreement on the status of a philosophy of nature. If, for instance, organism can for both of them be considered as an « analogon » of the absolute, it all depends on how they conceive this analogy: from a practical or from a theoretical point of view.

KEYWORDS : Fichte, Schelling, Naturphilosophie, organism, transcendental idealism

$$
* * *
$$

Dans son cours de l'été 1929 sur l'idéalisme allemand, Heidegger dresse le constat suivant à propos de la philosophie de Fichte, en vue d'établir un contraste avec Schelling.

Le système de la doctrine de la science embrasse seulement le comportement théorique et le comportement pratique; le comportement théorique en général et le comportement 'pratique' dans l'exhaustion de l'acte de poser comme tel et de l'acte d'autoposition (par quoi doit se solder, à terme, l'ensemble de ce 
comportement). La nature dans sa teneur propre n'a pas de place, et l'art, cette manière fondamentale d'agir et d'entrer en relation avec le monde, n'est pas là .

Ce constat de l'absence de préoccupations de la part de Fichte pour la nature et pour l'art, deux thèmes que Heidegger associe explicitement à la troisième Critique de Kant, est en partie faux, en partie vrai. Le constat est inexact s'il signifie, par exemple, que l'art n'a pas de « place » dans l'architectonique de la philosophie fichtéenne. En effet, on est autorisé à faire valoir non seulement qu'une place est réservée à l'esthétique dans la doctrine de la science, mais aussi que Fichte a fourni une ébauche assez complète de ce qu'aurait comporté cette partie du système. Seulement voilà : ces grandes lignes de l'esthétique fichtéenne ne sont tout simplement « pas là »-- et en ceci il faut donner raison à Heidegger - pour l'excellente raison que Fichte n'a pas eu le loisir ou encore n'a pas ressenti l'urgence de les développer en détail et de les publier de son vivant. La reconstruction de cette discipline doit donc être faite à l'aide des manuscrits personnels de Fichte et des notes de cours qui ont été publiés à titre posthume ${ }^{2}$.

Or il en va de même de la philosophie fichtéenne de la nature. A l'époque où Heidegger prend la parole, la base textuelle qui pourrait servir à cautionner la présence chez Fichte d'une approche systématique de la nature est encore très mince. Entre-temps, toutefois, l'inventaire des matériaux pour la reconstruction de cette philosophie de la nature a été établi par Reinhard Lauth dans son ouvrage sur La théorie transcendantale de la nature chez Fichte dans lequel il met à contribution le vaste corpus d'inédits publiés depuis 1962 dans l'édition de l'Académie bavaroise des sciences ${ }^{3}$. C'est donc dire qu'on ne peut plus se contenter tout simplement de s'interroger sur la manière dont Schelling viendrait ici combler une 'lacune' chez Fichte, comme Heidegger à son époque est contraint de le faire. Nous sommes désormais en mesure de procéder à une comparaison, voire à une confrontation de leur philosophie de la nature respective.

\footnotetext{
${ }^{1}$ Martin Heidegger, Der deutsche Idealismus (Fichte, Schelling, Hegel) und die philosophische Problemlage der Gegenwart, Gesamtausgabe, t. 28, Francfort-sur-le-Main, Klostermann, 1997, p. 184, cf. 185.

${ }^{2}$ Voir à ce sujet Ives Radrizzani, « De l'esthétique du jugement à l'esthétique de l'imagination, ou de la révolution copernicienne opérée par Fichte en matière d'esthétique », dans S. Trottein, dir., L'esthétique naît-elle au XVIIIe siècle?, Paris, PUF, 2000, p. 135-156; notre contribution « L'esthétique a-t-elle une place dans la philosophie de Fichte? », Les cahiers de philosophie, numéro hors série (1995), Lille, p. 181202.

${ }_{3}^{3}$ Reinhard Lauth, Die transzendentale Naturlehre Fichtes nach den Prinzipien der Wissenschaftslehre, Hambourg, Meiner, 1984.
} 
Mais avant toute chose, il convient de s'arrêter sur la valeur symptomatique de ces deux carences dans l'œuvre publiée de Fichte en regard du système global de la doctrine de la science. Et en ce sens le diagnostic de Heidegger conserve sa pertinence : le fait que certains volets du système soient laissés dans l'ombre est éloquent par lui-même. En effet, pendant que Schelling s'affairait à rédiger les Idées pour une philosophie de la nature (1797) et L'âme du monde (1798), Fichte complétait la composante pratique de son entreprise en publiant son Fondement du droit naturel (1796-1797) et son Système de l'éthique (1798). Cela ne signifie pas pour autant que Fichte se désintéresse du volet théorique : au tournant du siècle il nourrit encore le projet d'élaborer pour son propre compte une philosophie de la nature, dont on ne retrouvera d'ailleurs pas la trace ${ }^{4}$. Il s'agit bien là pourtant d'une exigence interne au système. La « Division » d'ensemble annexée à la Doctrine de la science nova methodo fait état, en effet, d'une partie théorique dont l'objet est la nature, celle-ci étant considérée tant d'après ses « lois organiques » que ses « lois mécaniques ${ }^{5} »$. Il y a donc une place chez Fichte pour une approche théorique de la nature. Et si l'on s'en tient à la nature organique, seul aspect sous lequel nous allons établir la confrontation dans ce qui suit, il faut bien avouer que Schelling par ses contributions en ce domaine n'innove en rien, puisque d'emblée l'écrit programmatique de 1794 Sur le concept de la doctrine de la science... prévoyait des développements sur la « légalité de la nature en sa liberté ${ }^{\star} »$. Ce qui rend encore plus évidente l'absence d'une publication chez Fichte spécifiquement consacrée à ces

\footnotetext{
${ }^{4}$ Lettre de Fichte à Schelling du 3 octobre 1800, dans Fichte/Schelling, Briefwechsel, Francfort-sur-leMain, Suhrkamp, 1968, p. 90; trad. fr. M. Bienenstock, Fichte/Schelling, Correspondance, Paris, PUF, 1991, p. 80.

On peut en dire autant d'ailleurs de Schelling : en 1796, il se proposait pour sa part d'écrire un « système de l'éthique », lequel ne verra jamais le jour. Cf. la lettre de Schelling à Niethammer du 22 janvier 1796, dans M. Frank et G. Kurz, dir., Materialien zu Schellings philosophischen Anfängen, Francfort, Suhrkamp, 1975, p. 141. Voir également F. W. J. Schelling, Vom Ich als Prinzip der Philosophie oder über das Unbedingte im menschlichen Wissen (1795), Sämmtliche Werke, Partie I, t. 1, édition de K. F. A. Schelling publiée à Stuttgart chez Cotta, 1856-1861 (cité ci-après $S W \mathrm{I} / 1$ etc.), p. 159; trad. fr. J.-F. Courtine, Du Moi comme principe de la philosophie ou sur l'inconditionné dans le savoir humain, dans Schelling, Premiers écrits (1794-1795), Paris, PUF, 1987, p. 59.

5 J. G. Fichte, Wissenschaftslehre nova methodo, éd. E. Fuchs, Hambourg, Meiner, 1994, p. 241; trad. fr. I. Thomas-Fogiel, Doctrine de la science nova methodo, Paris, Le livre de poche, 2000, p. 332.

${ }^{6}$ Fichte, Über den Begriff der Wissenschaftslehre oder der sogenannten Philosophie, Gesamtausgabe der Bayerischen Akademie der Wissenschaften, Partie I, t. 1, édité par R. Lauth et H. Jacob, Stuttgart, Frommann-Holzboog, à partir de 1962 (cité ci-après GA I/1 etc.), p. 151; trad. fr. L. Ferry et A. Renaut, Sur le concept de la doctrine de la science ou de ce que l'on appelle philosophie, dans Fichte, Essais philosophiques choisis (1794-1795), Paris, Vrin, 1984, p. 70.
} 
questions. Par delà les impératifs de la division du travail en vue de la complétion du système de la doctrine de la science, impératifs auxquels se soumettent de bon gré Fichte et Schelling, des intérêts philosophiques divergents s'annoncent qui se manifesteront avec éclat au moment de la rupture de leurs relations en 1801.

Dans ce qui suit, nous allons restreindre l'examen des rapports entre les philosophies de la nature de Fichte et de Schelling à la période qui précède la rupture, c'est-à-dire à cette période où l'on note d'intéressants recoupements et d'évidentes convergences, et où simultanément les germes du différend à venir commencent à se développer ${ }^{7}$. Nous procéderons aussi en deux temps. Dans la première partie, nous aurons l'occasion de constater une remarquable unité de vues entre les deux penseurs en regard des thèmes et des principales thèses touchant la philosophie de la nature organique. Cette coïncidence des thématiques nous offre au demeurant la possibilité de porter notre regard sur le sort réservé par Schelling à l'approche philosophique privilégiée par Fichte et qui deviendra plus tard précisément objet de litige : l'idéalisme transcendantal. En tout état de cause, Fichte se sentira encore en mesure de confier à Schelling en 1800 que sa saisie de l'idéalisme transcendantal adopte sans doute des allures de génialité, mais qu'elle n'en demeure pas moins pour l'essentiel juste (richtig) et pertinente $\left(\right.$ treffend $\left.{ }^{8}\right)$, ce que nous serons en mesure de confirmer à la lumière des Idées pour une philosophie de la nature de Schelling, ouvrage que Fichte qualifie de « remarquable ». Quant à la seconde partie, elle vise à anticiper le débat qui aura lieu au tournant du siècle en mettant en évidence la divergence des motivations philosophiques de Fichte et de Schelling. Celles-ci sont évidemment diffuses dans leurs textes, si bien qu'il y a lieu d'établir la comparaison de leur conception de la nature organique en choisissant un dénominateur commun. Or, dans la mesure où il est notoire que la Critique de la faculté de juger constitue pour chacun d'eux un pôle de référence tout à fait crucial, nous allons comparer leur lecture de cette œuvre en commençant par distinguer ce qui pour chacun des deux auteurs en constitue la porte d'entrée. Aussi pour

\footnotetext{
7 Nous empruntons à Marie-Christine Challiol-Gillet la désignation « première philosophie de la nature » de Schelling. Elle caractérise la période au cours laquelle la philosophie de la nature s'inscrit encore dans le sillage de l'idéalisme transcendantal fichtéen. Voir son Schelling, Paris, PUF, 1996, p. 11.

${ }^{8}$ Lettre de Fichte à Schelling du 3 octobre 1800, Briefwechsel, p. 89; trad. fr., p. 80. Voir aussi Fichte, GA III/2, 347. Fichte emploie le mot genialisch, dans lequel il n'est pas interdit de déceler une certaine ironie, à moins d'y voir carrément une mise en garde.
} 
Fichte est-ce l'Introduction qui offre le défi le plus redoutable, celui de solutionner le problème de l'inscription de la liberté dans le monde sensible, alors que pour Schelling la voie d'accès par excellence à cette œuvre se trouve dans le fameux $\S 76$. Kant y développe, comme on sait, l'idée d'un entendement intuitif qui, aux yeux de Schelling, illustre d'excellente manière la façon dont il faut concevoir l'absolu. « ... sans doute jamais autant de pensées profondes, commente Schelling, n'ont été exposées et en si peu de pages que dans le $\S 76$ de la Critique de la faculté de juger ${ }^{9}$ ».

\section{I - La philosophie transcendantale : une convergence de vues}

a) L'organisme universel

Schelling fait paraître en 1798 L'Áme du Monde avec le sous-titre suivant : « Une hypothèse de physique supérieure pour expliquer l'organisme universel ». Or, lorsque l'on prend connaissance du contenu de cette œuvre, on réalise bientôt qu'il n'y est pas question uniquement de nature organique, mais aussi de nature inorganique. Reste que le sous-titre demeure pertinent pour l'ensemble de l'œuvre dans la mesure où l' « organisme universel » en question ne renvoie pas à la nature comme à un grand animal, ainsi que pourrait le laisser croire l'expression « âme du monde». En fait, Schelling se réfère à cette expression comme à une tournure « archaïque » et « poétique » qui n'a ici tout au plus que valeur de métaphore ${ }^{10}$. C'est l'organisme universel qui constitue en vérité la visée de l'ouvrage. Or, le monde ainsi conçu comme totalité organique n'exclut pas la nature inorganique, laquelle est au contraire partie intégrante du tout, dans la mesure où elle entre en interrelation étroite avec la nature organique. L'action réciproque qui unit l'inorganique à l'organique n'est donc pas purement mécanique, mais est envisagée comme une interaction de type organique, close sur elle-même et formant une totalité englobante.

Il faut toutefois le dire d'emblée, Fichte souscrit sans réserves à cette conception de la nature prise globalement. La thèse est énoncée en toutes lettres au $\S 8$ du Système

${ }^{9}$ Schelling, Vom Ich, SW I/1, 122 note; trad. fr., p. 147.

${ }^{10}$ Schelling, Von der Weltseele, $S W \mathrm{I} / 2,347,569$. 
de l'éthique paru la même année que l'ouvrage de Schelling : "La nature en général est... un tout organique et est posée comme telle ${ }^{11}$.» Or, la question est la suivante : s'agit-il d'une conception propre à Fichte ou bien la tient-il de Schelling? On sait en outre que ce dernier a déjà fait état de la thèse de l'organisme universel dans ses Abhandlungen publiées en 1796-1797. Doit-on conclure ici à une influence de Schelling sur Fichte ${ }^{12}$ ? A considérer les choses de plus près, il faut écarter cette conjecture puisque Fichte était déjà parvenu par lui-même à cette conception dans ses premières ébauches de la doctrine de la science. En effet, le manuscrit intitulé Philosophie pratique, qui fait suite aux Méditations personnelles sur la philosophie élémentaire et qui a été rédigé à 1'hiver 1794, fait d'emblée mention de la nature comme totalité organique ${ }^{13}$. En vérité, l'interdépendance « organique » des parties d'un tout traduit à souhait l'impératif du système auquel se soumet la doctrine de la science et qui doit aussi être valable pour la philosophie de la nature ${ }^{14}$.

Le constat que Fichte et Schelling partagent cette prémisse philosophique fondamentale nous invite à scruter les textes plus attentivement pour réaliser, avec stupéfaction, que les deux auteurs ont en commun un grand nombre de positions en regard de la philosophie de la nature. Ainsi les développements de L'Âme du Monde nous apprennent que le principe d'organisation est présent dans l'ensemble de l'univers (allgemeinverbreitet), même s'il n'est pas partout actualisé ${ }^{15}$. Or, pour Fichte, les choses ne se présentent pas de manière différente. Le $\S 8$ du Système de l'éthique révèle à son tour qu'il y a une tendance (Trieb) à l'organisation répandue à l'échelle de la nature

\footnotetext{
${ }^{11}$ Fichte, System der Sittenlehre nach den Prinzipien der Wissenschaftslehre, Fichtes Werke, t. IV, édition I. H. Fichte, Berlin, Veit \& Comp., 1845-1846 (cité ci-après Werke I etc.), p. 115; trad. fr. P. Naulin, Le système de l'éthique d'après les principes de la doctrine de la science, Paris, PUF, 1986, p. 111. Cf. W. Schmied-Kowarzik, « Das Problem der Natur. Nähe und Differenz Fichtes und Schellings », FichteStudien, vol. 12 (1997), p. 226.

${ }^{12}$ Schelling, Abhandlungen zur Erläuterung des Idealismus der Wissenschaftslehre, SW I/1, 386.

${ }^{13}$ Fichte, Practische Philosophie, GA II/3, 247, 257 : « La matière inorganique devient aussi organe dans ce système, c'est-à-dire qu'elle devient une partie de l'univers organisé ». Cf. Grundlage des Naturrechts nach den Prinzipien der Wissenschaftslehre, Werke III, 209 ; trad. fr. A. Renaut, Fondement du droit naturel selon les principes de la doctrine de la science, Paris, PUF, 1984, p. 220. Wissenschaftslehre nova methodo, p. 238; trad. fr., p. 329.

${ }^{14}$ Fichte, Über den Begriff der Wissenschaftslehre..., Werke I, 38; trad. fr., p. 29. Voir également R. Lauth, Die transzendentale Naturlehre Fichtes..., p. 131. Schelling, Ideen zu einer Philosophie der Natur, $S W \mathrm{I} / 2,54$ : « ... la nature devient une ligne courbe, qui se replie sur elle-même, un système clos sur luimême... ». Von der Weltseele, $S W \mathrm{I} / 2,381$.

${ }^{15}$ Schelling, Von der Weltseele, SW I/2, 567.
} 
entière $^{16}$. Ce qui vient corroborer la thèse schellingienne selon laquelle, bien que toute matière dans l'univers ne soit pas d'emblée organisée, la nature n'en forme pas moins un organisme universel. De la même manière, lorsqu'on le considère à l'échelle de l'organisme individuel, ce principe vient régir l'ensemble des parties d'une telle organisation : il est encore une fois présent partout. Ainsi, puisque chacune des parties d'un corps organisé ne peut maintenir son existence que grâce au concours des autres organes, chaque partie constitutive de l'organisme, si infime soit-elle, témoigne à sa manière de l'ensemble et porte la marque de l'individualité de l'organisme en question. En d'autres mots, chaque partie reflète le tout de l'organisme. À ce sujet, Fichte et Schelling sont à nouveau parfaitement à l'unisson, si bien que pour eux, tant du point de vue de la partie que du tout, le principe d'organisation est omniprésent et remplit la même fonction $^{17}$.

Ils s'entendent également sur la nature de ce principe, dans la mesure où celui-ci s'avère être immanent à la nature elle-même. Ce qui signifie que la nature n'est pas seulement un tout 'organisé', mais qu'elle est en elle-même l'instance organisatrice. On découvre du reste tant chez Fichte que chez Schelling l'emploi du participe présent « organisant » (organisierend) pour désigner cette immanence du principe producteur. Dans les Plattner-Vorlesungen de Fichte, l'argument contre une vision purement mécaniste de la nature prend la forme suivante :

L'une des principales erreurs de la physique jusqu'à présent tient au fait que l'on considère toujours la nature comme étant simplement organisée, et non pas comme organisante. Sous ce dernier aspect, on découvre que la force en présence produit elle-même et pour elle- même les conditions de ses effets ${ }^{18}$.

La dynamique à l'œuvre au sein de l'organisme n'est donc pas tributaire d'une impulsion extérieure; elle fait preuve à cet égard d'une forme d'autodétermination. L'organisme est en ce sens ce que Kant appelait une « fin naturelle » : si chaque organe rend possible le tout qui en retour rend lui-même l'organe possible, nous assistons là à une finalité qui

\footnotetext{
16 Fichte, System der Sittenlehre, Werke IV, 121; trad. fr., p. 116.

17 Fichte, Vorlesungen über Logik und Metaphysik, GA IV/1, 389; System der Sittenlehre, Werke IV, $114-$ 115; trad. fr. p. 111; Grundlage des Naturrechts, Werke III, 61 ; trad. fr., p. 76. Schelling, Von der Weltseele, $S W \mathrm{I} / 2,517,519-520$.

18 Fichte,Vorlesungen über Logik und Metaphysik, GA IV/1, 394. Voir également Wissenschaftslehre nova methodo, p. 237; trad. fr., p. 329. Schelling, Von der Weltseele, SW I/2, 381; Erster Entwurf eines Systems der Naturphilosophie (1799), SW I/3, 17 note.
} 
ne peut en aucun cas être imposée du dehors. A titre de produit naturel, l'organisme doit être envisagé uniquement selon la finalité interne. Fichte et Schelling en conviennent : c'est à cette seule condition que l'on demeure fidèle à ce qui fait la spécificité de la finalité naturelle. Car, dès lors que cette finalité est conçue comme relative, c'est-à-dire du moment qu'elle est rapportée à une intelligence extérieure (humaine ou divine), les données du problème sont faussées ${ }^{19}$. Ainsi le recours à une cause transcendante, tel un architecte du monde (Weltbaumeister), ne peut que reproduire le modèle de l'artisan extérieur à son produit et rabaisser la nature à une simple machine, sans compter qu'il prive cet architecte de sa dimension proprement divine, lui conférant un entendement discursif comme l'est celui de l'être humain ${ }^{20}$.

La convergence de vues entre Fichte et Schelling se confirme encore lorsque l'on porte attention à la terminologie qu'ils emploient pour désigner la dynamique à l'œuvre dans la nature organique. Ils ont en effet tous deux recours - s'inspirant en cela de Kant, il est vrai - au concept de Bildungstrieb introduit par Blumenbach, et que l'on pourrait traduire par « tendance formatrice ${ }^{21} »$. Outre le fait que l'expression comporte la composante -trieb (tendance), capitale pour la pensée de Fichte comme nous aurons l'occasion d'y revenir ci-dessous, ce terme dans son acception scientifique offre l'avantage d'avoir été formé précisément pour aller à l'encontre des explications mécanistes de l'organisme. Bien sûr, une simple expression ne constitue pas en ellemême une explication et Schelling conserve malgré tout quelques réserves relativement à l'usage de ce terme, dans la mesure où la tendance formatrice à l'œuvre dans l'organisme présuppose déjà, selon la théorie même de Blumenbach, la présence de matière organisée. Quoi qu'il en soit, Schelling, tout comme Fichte, préfère cette expression à celle de Lebenskraft, puisque le concept de 'force' ne peut relever que du domaine de l'inorganique et que, pour cette raison, la force est toujours en elle-même « morte » et

\footnotetext{
${ }^{19}$ Fichte, System der Sittenlehre, Werke IV, 128-129; trad. fr., p. 123-124 : « Il n’y a dans la nature qu'une finalité interne et non pas une finalité relative. Cette dernière n'est engendrée que par les fins arbitraires qu'un être libre a la faculté de poser pour soi et aussi, pour une part, de réaliser dans les objets de la nature ». Cf. E. Fleischmann, « Science et intuition dans la Naturphilosophie de Schelling », dans G. PlantyBonjour, dir., Actualité de Schelling, Paris, Vrin, 1979, p. 55; Terry Pinckard, German Philosophy 17601860. The Legacy of Idealism, Cambridge, Cambridge University Press, 2002, p. 181.

${ }^{20}$ Fichte, System der Sittenlehre, Werke IV, 119; trad. fr., p. 115. Schelling, Ideen, SW I/2, 45.

21 Fichte, Practische Philosophie, GA II/3, 256. System der Sittenlehre, Werke IV, 121; trad. fr., p. 116. Schelling, Abhandlungen, SW I/1, 386. Von der Weltseele, SW I/2, 499, 522, 527.
} 
demande à être sollicitée de l'extérieur, ce qui vient en contradiction avec la finalité interne de l'organisme vivant ${ }^{22}$. Le Bildungstrieb, quant à lui, traduit très bien cette dynamique interne à l'organisme présidant à son développement progressif. Il confère de la sorte une part active à la nature dans ses productions. Le terme s'inscrit d'ailleurs chez Blumenbach dans une théorie épigénétique visant à faire obstacle à la théorie biologique de la préformation, selon laquelle l'individu à naître est d'entrée de jeu entièrement constitué, quoique sous forme microscopique, dans les semences des premiers individus de l'espèce, tels qu'ils sont sortis des mains du Créateur. Or, de même que Fichte et Schelling s'opposent vigoureusement à cette variante épistémologique du préformationnisme qu'est la théorie des idées innées ${ }^{23}$, de même dans le champ des sciences de la nature refusent-ils cette théorie que l'on appelle aussi de l' « emboîtement » ou de l' « involution », et qui ne concède aucun rôle véritable à la nature dans la production des individus des diverses espèces ${ }^{24}$.

Il convient toutefois de souligner que le recours à la tendance formatrice à titre de principe organisateur dans la nature n'implique pas que Fichte et Schelling soient prêts à laisser libre cours à un processus de formation à outrance, privé de toutes balises. Au contraire, tous deux considèrent qu'il existe une échelle fixe des espèces, échelle issue d'une stabilisation du processus de formation. En fait, la tendance formatrice, après avoir présidé au développement complet de l'individu d'une espèce, se tourne vers la survie de l'espèce sous la forme de la reproduction sexuée.

Dès le moment où la sexualité est présente, la nature ne produit plus par ce moyen de nouveaux individus, car la partie de la tendance formatrice de la nature qui serait requise pour ce faire, n'est plus répandue dans la nature brute, mais elle est dans ces produits; la force continue à agir dans les produits achevés, elle en produit de nouveaux [exemplaires] et conserve ceux qui existent déjà ${ }^{25}$.

\footnotetext{
22 Schelling, Ideen, SW I/2, 49-50; Von der Weltseele, SW I/2, 566; Erster Entwurf, SW I/3, 152. Voir sous ce rapport Wolfgang Wieland, « Die Anfänge der Philosophie Schellings und die Frage nach der Natur », dans M. Frank et G. Kurz, dir., Materialien zu Schellings philosophischen Anfängen, p. 278, note 40. Fichte, Vorlesungen über Logik und Metaphysik, GA IV/1, 390.

${ }^{23}$ Fichte, Vorlesungen über Logik und Metaphysik, GA IV/1, 354. Schelling, Abhandlungen, SW I/1, 351 n, 392, 426. Cf. Marc Maesschalck, « Construction et réduction. La méthode des philosophies de la nature chez Fichte et Schelling entre 1800 et 1806 », Les études philosophiques, nº 4 (1997), p. 459.

24 Schelling, Von der Weltseele, SW I/2, 528.

25 Fichte, Vorlesungen über Logik und Metaphysik, GA IV/1, 410, cf. 398. Schelling, Ideen, SW I/2, 40; Abhandlungen, $S W \mathrm{I} / 1,387$. Voir à ce sujet Jörg Jantzen, « Die Philosophie der Natur », dans H. J. Sandkühler, dir., F.W.J. Schelling, Stuttgart/Weimar, Metzler, 1998, p. 88, 97. Sur le rôle joué ici par Herder et Kielmeyer, voir aussi Thomas Bach, « Kielmeyer als 'Vater der Naturphilosophie'?
} 
En somme, pas plus chez Fichte que chez Schelling il n'est possible de faire intervenir le concept d'évolution au sens darwinien du terme. Le Bildungstrieb a trouvé son point d'équilibre dans les espèces actuelles.

Ce rapide tour d'horizon nous permet de constater une remarquable coïncidence entre l'approche philosophique de la nature de Fichte et celle de Schelling. Plutôt que de chercher à découvrir les traces d'influences réciproques entre les deux auteurs, nous nous sommes bornés à souligner les points de rencontre en donnant la priorité aux développements de Fichte puisque, en l'absence de grands traités systématiques comme ceux de Schelling, la théorie fichtéenne demande à être reconstruite à l'aide des manuscrits et des diverses allusions dans les écrits publiés. Nous avons consulté, pour ce faire, l'ébauche cursive d'une philosophie de la nature en sa « libre légalité » contenue dans le manuscrit Philosophie pratique (1794), les leçons sur la Logique et la métaphysique (1797) dont les thèmes sont, du reste, dictés à Fichte par le recueil d'aphorismes de Plattner, les dernières sections de la Doctrine de la science nova methodo et enfin le $\S 8$ du Système de l'Éthique, dont le traitement consacré à la nature est évidemment tributaire de l'architectonique de ce livre. On doit, en dernière analyse, attribuer cette convergence de vues, qui s'est dégagée des textes, au fait que le discours des deux auteurs s'enracine dans la doctrine de la science. Aussi n'est-ce pas par complaisance que Fichte affirme que Schelling a bien saisi le sens et la portée de sa philosophie. À la lecture des Idées et de L'Âme du Monde, il ne peut que voir son jugement confirmé, tant il est vrai que la perspective philosophique ultime de ces œuvres consacrées à l'étude de la nature demeure l'idéalisme transcendantal : « ...nous pouvons remarquer, souligne Xavier Tilliette, que, dans ces deux ouvrages, la philosophie de la nature reste grosso modo une application de la philosophie transcendantale ${ }^{26} \gg$. Dans la section qui suit, nous allons présenter un exemple tiré des Idées en cherchant à montrer à quel point la philosophie schellingienne de la nature n'entend encore nullement rompre les ponts avec la pensée de Fichte, en sorte que la véritable émancipation de la Naturphilosophie ne se produira que plus tard. Cette étape ultérieure de l'itinéraire de

Anmerkungen zu seiner Rezeption im deutschen Idealismus », dans K. T. Kanz, dir., Philosophie des Organischen in der Goethezeit, Stuttgart, Franz Steiner Verlag, 1994, p. 243.

26 Xavier Tilliette, Schelling, une philosophie en devenir I, Paris, Vrin, 1969, p. 172. 
Schelling ne retiendra pas notre attention ici, nous l'avons déjà dit. Nous nous bornerons à souligner dans la seconde partie de ce travail quelques notes discordantes de la première philosophie de la nature schellingienne.

a) Schelling : l'argument anti-dogmatique des Idées

La première partie des Idées publiée en 1797 ne connaîtra pas de suite, si bien que dans ses développements cette œuvre ne touche pas la physiologie, que Schelling avait réservée pour la seconde partie. Par contre, son intérêt pour les questions relatives à la nature organique est très manifeste dans l'Introduction de l'œuvre, qu'il a vraisemblablement rédigée après avoir écrit les deux livres composant la première partie et qui peut-être vue comme une transition vers $L$ 'Ame $d u$ Monde, à laquelle il commencera à travailler au plus tard en septembre $1797^{27}$. C'est toutefois dans un contexte bien précis que le thème de l'organisme intervient dans cette Introduction. En effet, celle-ci ne se situe pas d'emblée sur le plan de la science de la nature, elle se veut au contraire éminemment philosophique dans la mesure où Schelling tient à y préciser le cadre théorique de ses recherches. Aussi la question qu'il pose d'entrée de jeu est-elle la question philosophique par excellence, celle précisément à laquelle Fichte à la même époque tente de répondre dans la Première introduction à la doctrine de la science, précisément la question de la possibilité de l'expérience. Alors que chez Fichte l'interrogation se formule ainsi : « quel est le fondement du système des représentations accompagnées du sentiment de nécessité ${ }^{28}$ ? », pour Schelling elle prend simplement la forme suivante : « comment un monde à l'extérieur de nous, comment une nature et avec elle l'expérience sont-ils possibles ${ }^{29} »$ ? Cette question avère en fait incontournable pour un ouvrage qui prétend jeter les bases d'une 'philosophie' de la nature. Or, Schelling ne tarde pas à prendre parti et laisse clairement entendre qu'il se réclame de l'idéalisme transcendantal. La méthode qu'il privilégie est « génétique » en ce qu'elle se propose de

\footnotetext{
27 Voir à ce sujet la préface de M. Durner à l'édition de l'Académie de Schelling, Ideen zu einer Philosophie der Natur, Werke I/5, Stuttgart, Frommann-Holzboog, 1994, p. 16-17.

${ }^{28}$ Fichte, Erste Einleitung in die Wissenschaftslehre, Werke I, 423; trad. fr. I. Thomas-Fogiel, dans Fichte, Nouvelle présentation de la doctrine de la science (1797-1798), Paris, Vrin, 1999, p. 99.

29 Schelling, Ideen, $S W \mathrm{I} / 2,12$.
} 
« déduire de la nature de notre... esprit la nécessité de la succession de nos représentations ${ }^{30} »$.

Toutefois, c'est par la voie de la polémique que Schelling dans cette Introduction choisit de prouver la vérité de l'idéalisme critique : il dénonce le dogmatisme et s'en prend en particulier à cette forme insidieuse de dogmatisme, qui tout en maintenant le caractère irréductible de la chose en soi retient du kantisme que les catégories de cause et d'effet, par exemple, sont simplement subjectives. Il vise en fait ici les « hommes de la philosophie kantienne » qui, alors qu'ils acceptent le statut a priori des catégories, soutiennent que les choses extérieures agissent sur le sujet. Dans les Lettres philosophiques sur le dogmatisme et le criticisme, Schelling qualifiait cette position de mauvais compromis (Mittelding) entre le dogmatisme et le criticisme. Il entend bien ici faire la preuve que cette approche éclectique est intenable ${ }^{31}$.

Or, à première vue, lorsque Schelling fait intervenir le thème de l'organisme au centre de la discussion, on pourrait être porté à croire qu'il fournit un argument supplémentaire au dogmatique. Il prétend en effet que contrairement aux autres « phénomènes » de la nature, l'organisme s'impose au sujet connaissant comme un véritable vis-à-vis, c'est-à-dire comme un « objet » qui représente une entité entièrement constituée, laquelle doit être acceptée telle quelle, dans son intégrité et son indépendance. Il semblerait donc que l'organisme vienne accentuer la césure déplorée par Schelling entre le sujet et l'objet, entre le sujet connaissant et la nature hors de lui, entre le Moi et la chose en soi. Toutefois, cette apparence est trompeuse car, à vrai dire, le thème de la nature organisée annonce, au contraire, la faillite du dogmatisme : «Quand vient le temps d'expliquer la finalité des produits organiques, soutient Schelling, le dogmatique se voit entièrement abandonné par son système $\mathrm{e}^{32} »$. Loin donc de constituer un argument sérieux aux mains du dogmatique, le défi philosophique posé par l'organisme est appelé à consacrer la supériorité de l'idéalisme transcendantal.

\footnotetext{
30 Schelling, Ideen, $S W \mathrm{I} / 2,35,39$.

31 Schelling, Ideen, SW I/2, 17 note. Philosophische Briefe über Dogmatismus und Kriticismus, SW I/1, 301; trad. fr. Lettres philosophiques sur le dogmatisme et le criticisme, dans Premiers écrits (1794-1795), p. 172. Parmi les kantiens visés ici, il faut certes compter le Reinhold de la « théorie de la faculté humaine de représentation », pris à parti dans une remarque de Schelling dépourvue de toute équivoque: l'argument qui consiste à dire que la chose en soi, bien qu'elle existe, n'est pas « représentable » s'avère irrecevable. Schelling, Ideen, $S W \mathrm{I} / 2,33$.

32 Schelling, Ideen, $S W \mathrm{I} / 2,41$.
} 
À l'intérieur du système hybride que défendent les kantiens en question, l'objet de la connaissance n'est toujours qu'un phénomène. C'est-à-dire que sa matière provient de la chose qui affecte, alors que sa forme est issue du sujet lui-même, qu'il s'agisse de la forme spatio-temporelle de l'intuition ou des concepts purs de l'entendement. Dans ces conditions, le découpage qu'opère le sujet connaissant à l'aide de ses concepts à l'intérieur des phénomènes est laissé à sa discrétion. Par exemple, grâce aux catégories de la quantité, il lui est possible de rassembler à sa guise divers éléments pour en former un tout, dont il assume lui-même l'unité et la cohérence. La forme (ici la quantité) et la matière sont deux instances indépendantes l'une de l'autre, en sorte que le découpage conceptuel demeure « arbitraire ».

Tant et aussi longtemps que vous rassemblez dans un nombre des choses qui sont séparées par l'espace, vous agissez librement; l'unité que vous leur donnez, c'est en fait vous qui à partir de vos pensées la transposez sur elles. Dans les choses elles-mêmes il n'y a pas de fondement [Grund] qui vous obligerait à les penser comme une. En revanche, que vous pensiez chaque plante comme un individu dans lequel tout concourt à une seule et unique fin, de ceci vous devez chercher le fondement dans la chose hors de vous ${ }^{33}$.

Ici se pose à nouveau la question introduite plus tôt : pourquoi l'organisme, en l'occurrence la plante ne reçoit-elle pas son unité du sujet? Pourquoi Schelling doit-il convenir que son principe se trouve hors du sujet, dans l'objet lui-même? Ce qui au premier abord, on l'a déjà dit, semble creuser plus profondément encore l'abîme entre le moi et le monde.

La réponse de Schelling peut se résumer ainsi : dans l'organisme, forme et matière sont absolument inséparables. Chaque partie est essentiellement tributaire des autres dans un réseau de relations qui constituent la forme de l'ensemble. Or, le fondement de cette forme, de cette unité qui assure la cohérence du tout doit être cherché dans le « concept ». C'est dire que les organismes ont ceci de particulier qu'ils possèdent leur concept à l'intérieur d'eux-mêmes. Nous n'avons dès lors plus affaire, comme c'était le cas des objets considérés comme "simples phénomènes », à cette indifférence et à cet arbitraire qui règnent entre forme et matière, entre le concept et son objet. Le concept et la matière sont dans le corps organique indissociables, sous peine de faire éclater l'individualité de l'objet : « ...ce concept réside dans [1'organisation] elle-même, il ne 
peut en être séparé; elle s'organise elle-même, elle n'est pas, par exemple, un ouvrage d'art, [car alors] son concept se situerait à l'extérieur d'elle dans l'entendement de l'artisan ${ }^{34} »$. On le voit, il est question ici de la finalité interne, et non de la finalité imposée de l'extérieur par l'artisan à son produit.

Le nerf de l'argument développé par Schelling est donc le suivant : la présence du concept, comme principe unificateur, à l'intérieur même de l'organisme ne signifie pas pour autant que l'objet en question émigre hors de la sphère du sujet. Bien au contraire, c'est l'objet lui-même qui est de la sorte rapatrié par le sujet, et ce, pour l'excellente raison qu'il n'y a de concept que pour un entendement. En dépit de l'autarcie dont il fait preuve dans le champ des phénomènes, l'organisme ne demeure toujours qu'un objet « pour moi », car une fin naturelle n'est rien sans le « jugement » porté sur elle et son concept $^{35}$. «Cette unité, soutient Schelling, n'est là qu'en rapport à un être intuitionnant et réfléchissant ${ }^{36}$. » En d'autres termes, la nature organique interpelle directement l'esprit, elle n'a de sens que pour lui, si bien que l'on est désormais dispensé de cette chimère qu'est la chose en soi. Le cas privilégié de l'organisme prouve, si besoin en était, qu'il n'y a pas de matière provenant de l'affection du sujet par une chose purement étrangère, par ce non-moi hypostasié que représente la chose en soi. La pénétration du concept au plus intime de la matière au sein de l'organisme plaide plutôt pour l'appartenance mutuelle des deux composantes du phénomène, si bien que la matière s'avère dépendre elle-même du moi.

Dans ces conditions, il est possible de faire une lecture scrupuleusement fichtéenne de cette phrase tirée de l'Introduction aux Idées : « Le système de la nature est en même temps le système de notre esprit ${ }^{37}$ ». Elle annonce d'ailleurs le point culminant de cette Introduction, souvent cité, qui conformément à la lecture que nous venons d'en faire, peut être entendu dans le même sens : «Ici donc, dans l'identité absolue de l'esprit en nous et de la nature hors de nous, doit se résoudre le problème : comment est possible

${ }^{33}$ Schelling, Ideen, $S W \mathrm{I} / 2,43$, cf. 40, 41.

${ }^{34}$ Schelling, Ideen, $S W \mathrm{I} / 2,41$; Von der Weltseele, $S W \mathrm{I} / 2,515-516$.

${ }^{35}$ Schelling, Ideen, $S W \mathrm{I} / 2,52$.

${ }^{36}$ Schelling, Ideen, $S W$ I/2, 42; 42-43: « Le concept de finalité ne peut naître que dans un entendement et ce n'est que par rapport à un tel entendement qu'une chose peut être dite finale [zweckmässig]. »

${ }^{37}$ Schelling, Ideen, $S W \mathrm{I} / 2,39$. 
une nature hors de nous ${ }^{38}$ ? » En effet, il n'est nullement requis d'y voir l'anticipation de la philosophie de l'identité encore à venir, du moins tant et aussi longtemps que demeure en vigueur pour Schelling l'idéalisme transcendantal comme cadre de référence ultime. Preuve en est qu'au moment où il commence à officialiser l'autonomie de la Naturphilosophie vis-à-vis de la philosophie transcendantale, soit vers 1799-1800, Schelling est encore en mesure de reconnaître la justesse du point de vue de la philosophie transcendantale en regard de la nature. Ainsi dans les sections du Système de l'idéalisme transcendantal (1800) consacrées à la « Déduction de l'organique », il admet que la nature organique n'est rien de plus qu'une transposition du moi, que le reflet de l'intelligence elle-même. « L'on peut dire que la nature organique fournit la preuve la plus visible de l'idéalisme transcendantal, car toute plante est un symbole de l'intelligence. » Et Schelling de poursuivre, un peu plus loin : «c'est l'intelligence ellemême qui à travers les labyrinthes et dédales de la nature organique cherche à se réverbérer comme productive ${ }^{39} »$. Dans la perspective ici retenue, cette façon de voir est conforme à l'esprit du fichtéanisme. D'ailleurs, le Fichte du Système de l'éthique n'hésite pas à défendre un argument similaire en établissant une distinction rigoureuse entre l'objet qui dans le champ de l'expérience a la valeur d'un simple tout idéal et celui qui se présente d'emblée comme un tout réel (reelles Ganze), à savoir l'organisme. Il reprend ainsi la distinction schellingienne entre le simple phénomène (blosse Erscheinung) et l'objet (Objekt). Bien que Fichte, pour sa part, n'en tire pas un argument contre le dogmatisme larvé des Kantiens - on sait que la Seconde introduction à la doctrine de la science développe sa propre stratégie contre le spectre de la chose en soi l'allure de l'exposé est très proche de celle que l'on trouve chez Schelling ${ }^{40}$. Dès que l'on fait face non plus à un agrégat, mais à cet authentique compositum qu'est

\footnotetext{
38 Schelling, Ideen, $S W \mathrm{I} / 2,56$.

39 Schelling, System des transzendentalen Idealismus, SW I/3, 490, 494; trad. fr. C. Dubois, Le système de l'idéalisme transcendantal, Louvain, Peters, 1978, p. 139, 142.

${ }^{40}$ Fichte, System der Sittenlehre, Werke IV, 116; trad. fr., p. 113: « Si ce tout doit devenir un composé, il faut que les parties elles-mêmes et, en vérité, précisément ces parties, se réunissent sans intervention de ma pensée pour constituer un tout. »; ibid. 120; trad. fr., p. 115: « Puis, une intelligence peut bien relier et rassembler sans cesse, aussi longtemps qu'elle le veut, il en naît une agrégation, un alliage, mais jamais une fusion, ce qui suppose une force interne dans la nature elle-même. " Wissenschaftslehre nova methodo, p. 236; trad. fr., p. 327-328.
} 
l'organisme, il n'est plus question de procéder à un découpage de façon purement aléatoire, sans quoi c'est l'intégrité même de ce tout réel qui est mise en péril.

Il n'est sans doute pas interdit de voir poindre dans les Idées et L'Âme du Monde des indices annonciateurs de l'émancipation de la philosophie de la nature par rapport au cadre du transcendantalisme. Car en dépit des allégeances officielles de Schelling, il faut avouer que de proche en proche au fil des textes la nature « se positionne comme l'autre symétrique du $\mathrm{moi}^{41} \gg$. Nous devons à présent nous pencher sur ces indices. Mais une chose est certaine, nous sommes encore loin ici des thèses de Schelling qui prendront le contre-pied de l'argument des Idées que nous venons de présenter. Ainsi, par exemple, dans les Aphorismes sur la philosophie de la nature de 1806, il n'hésitera pas à partir en guerre contre ceux qui se montrent incapables de voir à l'œuvre dans la nature un concept, sans le rattacher aussitôt à une âme. Schelling plaidera en fait pour un idéalisme objectif, au sein duquel le « concept vivant» est réputé pouvoir subsister dans la nature par lui-même, sans le rapport à un entendement, c'est-à-dire sans devoir s'inscrire nécessairement dans un processus de réflexion consciente ${ }^{42}$ ! Comme nous allons le voir, les dissidences de Schelling par rapport à Fichte prennent dans les années 1790 une forme qui, pour instructive qu'elle soit, demeure beaucoup moins spectaculaire.

\section{II - L'organisme, trait d'union entre nature et liberté}

Par-delà le recoupement des thèmes, voire des thèses respectives de Fichte et de Schelling, il devient manifeste que les différences d'accentuation qui se font jour dans la première philosophie de la nature de Schelling sont imputables à des divergences qui touchent leur motivation philosophique profonde. Ainsi, faut-il y insister, les

\footnotetext{
${ }^{41}$ Miklos Vetö, De Kant à Schelling. Les deux voies de l'idéalisme allemand, tome I, Grenoble, J. Millon, 1998, p. 455.

${ }^{42}$ Schelling, Aphorismen über die Naturphilosophie (1806), SW I/7, 215 note; trad. fr. J.-F. Courtine et E. Martineau, Schelling, Euvres métaphysiques (1805-1821), Paris, Gallimard, 1980, p. 89-90 note: « La plupart des gens croient très bien comprendre qu'une âme ait des concepts, des représentations, etc.; mais qu'il puisse y avoir dans la nature des concepts vivants, qui ne sont rien d'autre que ces concepts mêmes qui ne peuvent être 'eus', mais subsistent pour soi et agissent en tant que tels, sans réflexion - voilà qui pour eux appartient non seulement aux choses incompréhensibles, mais même parfaitement inintelligibles... »
} 
préoccupations philosophiques de Fichte sont-elles d'abord et avant tout pratiques: il s'intéresse à la nature principalement en tant que lieu de la réalisation d'une action libre. En revanche, on décèle dans le texte de Schelling une attitude beaucoup plus contemplative, plus réceptive à la richesse du spectacle qu'offre la nature. D'où l'on peut conclure que la compréhension précise du processus d'engendrement des diverses formes dans la nature présente pour lui un intérêt spéculatif crucial, lequel n'est pas sans lien avec la manifestation de l'absolu. En fait, la différence entre Fichte et Schelling quant au statut de la nature renvoie aux modalités de cette manifestation de l'absolu. En procédant brièvement, il est en effet permis de résumer les deux positions de la manière suivante. Si chez Fichte l'intuition intellectuelle donne accès au sujet absolu, celui-ci se voit d'emblée placé sous l'injonction de la loi morale qui commande de réaliser la liberté afin d'atteindre, à la faveur d'une tâche infinie, le Moi comme Idée. En revanche, l'intuition intellectuelle telle que conçue par Schelling dévoile un Moi absolu qui s’apparente au départ à la substance spinoziste. L'absolu s'y manifeste donc comme un Être plutôt que comme un Devoir-être; d'où l'importance et la relative autonomie du volet théorique chez Schelling, comme nous aurons bientôt l'occasion de le constater ${ }^{43}$.

S'il est un dénominateur commun qui permet de diagnostiquer ces options philosophiques divergentes, c'est la Critique de la faculté de juger de Kant qui nous l'offre. En effet, nos deux auteurs ont en commun d'avoir nourri à un certain moment le projet de rédiger un commentaire de cette œuvre, ce qui prouve d'emblée l'intérêt qu'elle a éveillé chez eux. Que le projet de part et d'autre n'ait pas abouti demeure un problème secondaire, puisque leurs travaux ultérieurs constituent en fait une réponse à la provocation qu'ils ont ressentie au contact de la troisième Critique. Pour Fichte, cette provocation a eu lieu en tout début de carrière, au moment où il songeait à se tailler une place dans le monde philosophique par une publication qui aurait eu pour titre Essai d'analyse explicative de la Critique de la faculté de juger de Kant ${ }^{44}$. Cette œuvre demeurera, on le sait, un fragment inédit. En ce qui concerne Schelling, c'est dans une lettre du 22 janvier 1796 qu'il confie à Niethammer son dessein de rédiger un tel commentaire, en précisant qu'il veut mener l'entreprise « selon [ses] propres principes ».

${ }^{43}$ Cf. Alexis Philonenko, La liberté humaine dans la philosophie de Fichte, Paris, Vrin, 1980, p. 85-90. 
Le commentaire ne verra évidemment pas le jour, mais un passage capital des Abhandlungen (1796-1797) sur le concept d'organisation laisse entrevoir que cette préoccupation thématique demeure bien vivante chez lui et qu'elle trouvera un terrain d'élection dans ses grandes œuvres sur la philosophie de la nature ${ }^{45}$. Qu'il suffise d'ajouter pour l'instant qu'autant Fichte que Schelling s'intéressent à la faculté de juger téléologique pour cette raison qu'ils voient tous deux dans la finalité naturelle le moyen terme (Mittelglied) entre la nature et la liberté.

a) Fichte. L'inscription de la liberté dans le monde sensible

Il est bien sûr dommage que le manuscrit du commentaire littéral que Fichte se proposait de publier sur la troisième Critique ne couvre que l'Introduction et les seize premiers paragraphes de l'Analytique du beau. Mais ce fragment demeure tout de même fort révélateur dans la mesure où les remarques qui y sont faites sur l'Introduction de Kant concernent cette partie du livre que Fichte considère comme étant « la plus obscure ». C'est que Fichte prend très au sérieux l'urgence signalée d'entrée de jeu par Kant de combler le fameux « gouffre » qui sépare la législation de l'entendement, valable pour la nature sensible, de la législation de la raison, valable pour le monde suprasensible. Au terme de son entreprise critique, Kant s'interroge sur la possibilité pour la législation morale d'intervenir au sein du monde sensible. Le thème est bien connu. Cependant, comme le souligne Frederick Neuhouser, le texte de Kant comporte une ambiguïté foncière qui tient au fait que deux lectures sont possibles en regard de la nature précise de la finalité morale qui doit se réaliser dans le monde sensible ${ }^{46}$. Il peut s'agir soit du problème de la réalisation d'une action morale (libre) dans le monde, soit du problème du souverain bien, donc de la réalisation dans le sensible d'un bonheur qui soit proportionnel au mérite moral de l'agent. Quoi qu'il en soit de l'interprétation qui doit être considérée comme la bonne, il suffit de souligner que Fichte adopte la première des deux lectures

\footnotetext{
${ }^{44}$ Fichte, Versuch eines erklärenden Auszugs aus Kants Kritik der Urteilskraft (1790-1791), GA II/1, 318373. Voir à ce sujet Xavier Léon, Fichte et son temps I, Paris, A. Colin, 1954, p. 93.

${ }^{45}$ Schelling, Abhandlungen, $S W$ I/1, 386-388. Cf. Jochen Kirchhoff, Schelling, Reinbeck bei Hamburg, Rowohlt, 1994, p. 87.
} 
possibles, si bien qu'à ses yeux, au seuil de la troisième Critique, le problème de la liberté transcendantale qu'avait pourtant résolu la troisième Antinomie de la Critique de la raison pure, demeure entier. D'après Fichte, Kant n'a donc pas encore solutionné le problème de l'inscription de la liberté dans le monde sensible et, à envisager la solution que Fichte élaborera dans les années subséquentes, il y a tout lieu de croire qu'il considère insatisfaisante la timide tentative de rapprochement des deux extrêmes (mécanisme et finalité) contenue dans la Critique de la faculté de juger téléologique. Il demeure néanmoins attentif à l'indication de Kant de chercher les pistes de solution du côté des fins naturelles.

La solution achevée à laquelle parviendra Fichte est exposée dans le Système de l'éthique et elle revêt la forme d'une théorie des tendances (Triebe). La dynamique à l'œuvre dans la tendance s'oppose à celle que l'on trouve dans les causes mécaniques dans la mesure où la tendance exprime la finalité interne propre à l'organisme. C'est donc par ce moyen terme (Mittelglied) entre nature et liberté qu'est la tendance que passe la solution fichtéenne du problème de la réalisation concrète de l'action morale dans le monde. En raison des limites de cet exposé, il est impossible de retracer la genèse du concept de tendance chez Fichte. Il convient toutefois de souligner que, si c'est en 17901791 que Fichte rend compte, dans son commentaire, du problème non résolu de la liberté pratique dans la troisième Critique, c'est dans la seconde édition de l'Essai d'une critique de toute révélation (1793) qu'il élabore pour la première fois, au $\S 2$, la théorie des tendances qui allait marquer un net progrès sur la manière kantienne de poser et de résoudre le problème ${ }^{47}$. En effet, si Kant est en somme incapable de surmonter le clivage entre monde intelligible et monde sensible, la théorie de la tendance, parce qu'elle redéfinit la nature humaine en son essence unitaire, y parviendra.

\footnotetext{
46 Frederick Neuhouser, Fichte's Theory of Subjectivity, Cambridge, Cambridge University Press, 1990, p. 17-18. Voir également Miklos Vetö, De Kant à Schelling. Les deux voies de l'idéalisme allemand, tome I, p. 452-453.

47 Fichte, Versuch einer Kritik aller Offenbarung, Werke V, 16-39; trad. fr. J.-C. Goddard, Essai d'une critique de toute révélation, Paris, Vrin, 1988, p. 40-64. Voir l'Introduction de J.-C. Goddard, p. 26-32. De même J.-L. Vieillard-Baron, « Commentaire » de Fichte, Conférences sur la destination du savant, Paris, Vrin, 1994, p. 122-124. Sur le rôle joué par Reinhold dans l'adoption par Fichte du concept de « tendance », voir notre contribution intitulée « Fichtes Auseinandersetzung mit Reinhold im Jahre 1793. Die Trieblehre und das Problem der Freiheit », dans M. Bondeli et A. Lazzari, dir., Akten der zweiten internationalen Reinhold-Tagung, Bâle, Schwabe Verlag, à paraître.
} 
Rien d'étonnant, dès lors, que le concept de tendance développé dans le Système de l'éthique se trouve précisément dans les sections qui nous ont servi ci-dessus à exposer la conception fichtéenne de la nature organique. En fait, dans ces pages l'organisme n'est pas abordé pour lui-même, mais plutôt dans le but de cerner le rôle du corps propre : le moi fini, pour intervenir dans le monde, doit être doté d'un corps. D'où les développements de Fichte sur le concept d'organisme, considéré comme produit naturel. Le corps organique offre ceci de particulier que, tout en relevant du domaine de la nature, il échappe à la logique des causes mécaniques. Certes, l'argument est bien connu. Mais Fichte franchit une étape de plus en affirmant que l'être humain, envisagé comme simple produit de la nature, est animé par une tendance, un Trieb. « Ma nature est une tendance », voilà le point ultime auquel peut atteindre la nature en regard de l'homme ${ }^{48}$. En quoi, rétorquera-t-on dès lors, le concept de tendance fait-il progresser la résolution du problème de la liberté? En ce qu'il permet de repérer un point d'insertion de la finalité dans le monde matériel. La finalité de l'organisme, considéré d'un point de vue simplement animal, n'est bien sûr pas identique à la finalité de la loi morale, mais elle n'en ouvre pas moins une brèche, que Fichte déclare essentielle à l'accomplissement de la liberté dans le monde.

L'extrait suivant, tiré du $\S 8$ du Système de l'éthique se termine sur une remarque mise entre parenthèses, qu'il convient de lire au départ, car elle signale sans équivoque l'enjeu stratégique relié au thème de la tendance : « ...moyen terme (Mittelglied) dont nous aurons assurément le plus grand besoin pour expliquer la causalité de la liberté dans la nature. » Voyons à présent comment se présente la solution. Fichte introduit successivement trois strates - le mécanisme naturel, la tendance et la liberté absolue dont la seconde assume la fonction de chaînon intermédiaire.

D'après le concept du mécanisme de la nature, chaque chose est par une autre ce qu'elle est et manifeste son existence dans une troisième. D'après le concept de la tendance, chaque chose est par soi-même ce qu'elle est et manifeste son existence en agissant sur soi-même. Si donc on doit penser un être libre, ce concept, pris dans toute sa rigueur, sans la moindre modification, ne vaut sans doute pas comme concept de la tendance, mais comme concept de l'absolue liberté. La liberté est directement opposée au mécanisme de la nature et n'est en aucune manière déterminée par lui. Mais, s'il s'agit d'une tendance de la nature, il faut conserver le caractère de la nature en général, celui du mécanisme, à côté du

${ }^{48}$ Fichte, System der Sittenlehre, Werke IV, 110; trad. fr., p. 107. 
caractère de la tendance, donc réunir synthétiquement les deux; par là, nous obtiendrons un moyen terme [Mittelglied] entre la nature comme simple mécanisme (et aussi le concept de causalité) et la liberté comme opposé direct de tout mécanisme (et aussi le concept de substantialité ${ }^{49}$ )...

Il convient de noter qu'à cette étape de la discussion, Fichte n'est en mesure de livrer qu'une partie de la solution du problème de la liberté. Si la tendance est appelée à servir de Mittelglied, on n'aperçoit pas encore comment elle est reliée à la liberté humaine. Et pour cause, puisque la tendance n'intervient dans le passage cité qu'à titre de « tendance naturelle ». La suite du texte apportera donc d'autres éléments. Pour l'instant, il importe de remarquer que la tendance appartient à la nature prise dans son ensemble et que, en dépit de la relative autarcie de sa finalité interne, elle demeure tout de même liée à son environnement, c'est-à-dire à l'univers des causes mécaniques. Or, si Fichte peut la considérer comme une instance intermédiaire, comme un point d'intersection entre la nature et la liberté, c'est parce qu'elle rend possible l'introduction de la finalité dans la nature. Comment dès lors concevoir l'intervention d'une finalité non plus naturelle, mais morale?

Pour affronter ce second volet du problème, Fichte abandonne la perspective strictement naturaliste adoptée momentanément par le texte, pour considérer l'être humain dans son ensemble. La théorie de la tendance est en vérité capitale en ce qu'elle définit la totalité de la réalité humaine, y compris sa dimension nouménale. C'est dire que par-delà la dimension naturelle du Trieb, il y a une dimension suprasensible, que Fichte qualifie de tendance « pure », « spirituelle ${ }^{50} »$. Et ici il faut être attentif : il ne s'agit pas de deux tendances séparées qui, réunies, composeraient la nature humaine. Il n'y a au fond qu'une seule et unique tendance originaire (Urtrieb). Ce qui permet de compléter l'argumentation ci-dessus : la tendance naturelle (qui se manifeste par toutes sortes de besoins affectant le sens tant interne qu'externe) n'est pas la seule à animer l'être humain, elle se redouble d'un moment pur, faisant intervenir la conscience et l'intelligence, de sorte que la tendance s'élève par là irrémédiablement au-dessus de l'animalité. La liberté absolue propre à la tendance pure est désormais en mesure de

${ }^{49}$ Fichte, System der Sittenlehre, Werke IV, 115; trad. fr., p. 111-112.

${ }^{50}$ Fichte, System der Sittenlehre, Werke IV, 130; trad. fr., p. 126. 
régner sur l'ensemble de l'individu, constitué en dernière analyse d'une seule et unique tendance :

Ma tendance comme être naturel, ma tendance comme pur esprit, sont-ce là deux tendances différentes? Non : du point de vue transcendantal, toutes deux sont une seule et même tendance originaire qui constitue mon être. Elle est seulement considérée de deux points de vue différents. En effet, je suis sujet-objet et c'est dans l'identité et l'inséparabilité des deux que consiste mon être véritable ${ }^{51}$.

Avec l'introduction de la tendance originaire, Fichte se déleste de l'hypothèque du dualisme kantien dans ce qu'il a de rigide et parvient à fournir une description de l'action morale qui s'avère pour lui beaucoup plus satisfaisante. Ainsi, non seulement la liberté trouve-t-elle dans la tendance pure une instance susceptible d'incarner la volonté morale, mais la conception unifiée de l'agent moral comme tendance originaire permet à la volonté de canaliser et d'exploiter pour ses propres fins les « forces » qui relèvent de sa dimension naturelle. ${ }^{52}$ De même, le corps propre, considéré comme un produit simplement naturel, comme un organisme, peut-il désormais être pris systématiquement en charge en vue de répondre aux exigences de l'action morale. On le voit, la tendance naturelle sert véritablement de chaînon intermédiaire; mais ce chaînon entre mécanisme et liberté n'a au fond qu'un rôle transitoire, dicté par le Système de l'éthique. Il n'est que la porte d'entrée de la finalité morale dans la nature. La finalité animale reliée aux besoins de l'organisme est rapidement transcendée au profit de la liberté. Le corps propre, selon Fichte, doit devenir l'« instrument immédiat de la volonté », puisque le but du sujet moral est de s'élever au dessus de la naturalité pour la dominer ${ }^{53}$. L'optique adoptée ici par Fichte est donc clairement pratique et elle culmine dans une conception de la nature qu'il faut bien qualifier d'instrumentale. Toute différente, il va sans dire, de celle qui se dégage des textes de Schelling. Celui-ci serait-il en effet disposé à s'atteler à la tâche infinie de réaliser l'« indépendance absolue à l'égard de toute nature ${ }^{54} »$ ?

\footnotetext{
51 Fichte, System der Sittenlehre, Werke IV, 130; trad. fr., p. 125.

52 Fichte, System der Sittenlehre, Werke IV, 135; trad. fr., p. 130.

53 Fichte, System der Sittenlehre, Werke IV, 128; trad. fr., p. 123. Voir à ce sujet notre contribution « Le concept de nature chez Fichte », Cahiers de la revue de théologie et de philosophie, vol. 18 (1996), Suisse, numéro spécial La nature, p. 553-556.

54 Fichte, System der Sittenlehre, Werke IV, 131; trad. fr., p. 126.
} 
b) Schelling : l'organisme, expression de l'absolu

Les deux occurrences du concept de « moyen terme » (Mittelglied) que nous nous proposons d'étudier ici proviennent d'œuvres de Schelling qui s'inscrivent explicitement dans la ligne de la philosophie transcendantale: Du Moi comme principe de la philosophie (1795) et le Système de l'idéalisme transcendantal (1800). Sans doute la préface de cette dernière œuvre fait-elle la promotion de la philosophie de la nature à titre de vis-à-vis symétrique de la philosophie transcendantale, mais nous allons nous intéresser principalement aux passages qui traitent de la téléologie - puisque c'est elle qui constitue le chaînon intermédiaire - à l'intérieur de cet ouvrage. Aussi n'est-ce pas un hasard si le chapitre consacré aux principes téléologiques procède d'après « les principes de l'idéalisme transcendantal », sans égard donc à la nouvelle philosophie de la nature. En nous concentrant sur ces deux occurrences du Mittelglied, nous entendons montrer comment les divergences qui se font jour entre Fichte et Schelling à l'intérieur même de la philosophie transcendantale sont annonciatrices de l'autonomie croissante que Schelling reconnaît à la philosophie de la nature à partir de $1799^{55}$.

C'est dans une note apparaissant dans les dernières pages de $\mathrm{Du}$ Moi que la téléologie est présentée comme ce « moyen terme » réunissant philosophie théorique et philosophie pratique ${ }^{56}$. La mention est très brève et une lecture fichtéenne pourrait nous laisser croire que l'allusion implique que la philosophie théorique se trouve placée au service de la philosophie pratique ${ }^{57}$. Mais ce motif fichtéen classique n'est pas le seul que déploie le texte, notamment dans sa conclusion. C'est là en effet qu'est dévoilé l'angle d'approche privilégié par Schelling pour aborder les questions de téléologie. Qu'il suffise de rappeler ici le commentaire élogieux qu’y fait Schelling du $§ 76$ de la

\footnotetext{
55 Sur l'équilibre instable qui prévaut à cette époque entre la philosophie de la nature et l'idéalisme transcendantal, voir Paul Ziche, « Gehört das Ich zur Natur? Geistige und organische Natur in Schellings Naturphilosophie », Philosophisches Jahrbuch, vol. 108 (2001), p. 41-57. Birgit Sandkaulen-Bock, Ausgang vom Unbedingten, Über den Anfang in der Philosophie Schellings, Göttingen, Vandenhoeck \& Ruprecht, 1990, p. 93-98.

56 Schelling, Vom Ich, $S W$ I/1, 241 note 2; trad. fr., p. 147.

57 Cette dimension n'est d'ailleurs pas absente du texte puisque dans les pages précédentes Schelling avait concédé que « la philosophie théorique ne porte sur l'effectivité que pour permettre à la causalité pratique de trouver un domaine où cette présentation de la réalité infinie - la solution de sa tâche infinie - soit possible ». Schelling, Vom Ich, SW I/1, 238-239 note 1; trad. fr., p. 144.
} 
Critique de la faculté de juger ${ }^{58}$. Il ne fait pas de doute que Schelling est fasciné par le thème de l'entendement intuitif développé dans ce paragraphe. Kant y indique que pour un tel être inconditionné, il n'y a pas de différence entre la possibilité, la réalité et la nécessité. Le particulier et l'universel ne sont pas séparés, comme pour un entendement discursif, en sorte que la dimension de contingence propre, d'une part, à la réalisation du devoir moral dans le monde, d'autre part, à l'accord des causes mécaniques et de la finalité dans la nature organique, est entièrement absente. Dans sa conclusion, Schelling suit Kant de très près, d'assez près en tout cas pour reprendre l'analogie faite au $\S 76$ entre le cas de la finalité morale et celui de la téléologie naturelle ${ }^{59}$. La conclusion de $D u$ Moi établit donc, du point de vue de la finalité, un parallèle entre la philosophie pratique et la philosophie théorique, lesquelles représentent pour Schelling deux ordres bien distincts. Xavier Tilliette ne s'est d'ailleurs pas fait faute de le signaler : la juxtaposition des deux domaines rappelle Kant bien plus que Fichte. Cette remarque est tout à fait pertinente surtout quand on sait que la facture du $\S 76$ a servi de canevas à la conclusion de l'œuvre que nous examinons ici. À propos de la fin de Du Moi, Tilliette affirme en effet : « une tension mal résorbée subsiste pourtant...; philosophie théorique et philosophie pratique sont juxtaposées, disjointes, ce qui est d'ailleurs plus kantien que fichtéen $^{60} \gg$.

Xavier Tilliette ne saurait mieux dire. Et ce qui est proprement kantien dans les lignes que nous allons lire tient au fait que le texte, plutôt que de subordonner la philosophie théorique à la philosophie pratique, établit une simple analogie entre les deux domaines. À ce compte, force nous est de conclure que le moyen terme ne fonctionne

\footnotetext{
${ }^{58}$ Schelling, Vom Ich , SW I/1, 242 note 1; trad. fr., p. 147 note 'R'. Pour la place centrale qu'occupe aux yeux de Schelling la Critique de la faculté de juger téléologique dans l'œuvre de Kant, voir Schelling, Zur Geschichte der neueren Philosophie (1833-1834), SW I/10, 177; trad. fr. J.-F. Marquet, Contribution à l'histoire de la philosophie moderne, Paris, PUF, 1983, p. 196. Xavier Léon, suivi en cela par Xavier Tilliette, affirme que tout autant que Schelling, Fichte a été très tôt fasciné par la problématique du $§ 76$, alors qu'il recherchait un principe commun permettant de réunir le monde sensible et le monde intelligible. Pour étayer cette affirmation, il se réfère au passage rétrospectif de la Wissenschaftslehre de 1804 où Fichte reprend la métaphore kantienne de la « racine commune » entre l'entendement et la sensibilité. Or, cette métaphore est tirée, comme on le sait, de la première Critique, et rien ne prouve qu'elle soit reliée dans l'esprit de Fichte au $\S 76$. Cf. X. Léon, Fichte et son temps I, p. 341; X. Tilliette, Schelling, une philosophie en devenir I, p. 98. Fichte, Wissenschaftslehre (1804), Werke X, 104.

${ }_{59}$ Kant, Kritik der Urteilskraft, édition de l'Académie, t. V, p. 404; trad. fr. A. J.-L. Delamarre et coll., Critique de la faculté de juger, Paris, Gallimard, Coll. « Folio », 1985, p. 374. L'analogie est introduite au début du dernier alinéa par « De même on peut,...»

${ }^{60}$ X. Tilliette, Schelling, une philosophie en devenir I, p. 75.
} 
pas à la manière de Fichte. Si la finalité naturelle doit relier nature et liberté, c'est plutôt sur le mode de l'analogie qu'elle le fera. Or, l'analogie a ceci d'intéressant que la téléologie naturelle comporte le privilège d'illustrer de quelle manière ce produit de la nature qu'est l'organisme parvient à concilier simultanément et en lui-même mécanisme et technique, causes efficientes et causes finales. Évidemment, le sujet réfléchissant ne peut comprendre comment est possible cette fusion des deux types de causalité dans l'organisme, mais la simple présence dans l'expérience d'un produit naturel organisé l'oblige à se référer à un principe supérieur qui, même s'il demeure incompréhensible, doit se trouver à l'origine de cette union. Aussi le contraste est-il frappant lorsque l'on se tourne vers l'autre terme de l'analogie, la philosophie pratique. Ici l'union de la loi de la liberté et de la loi naturelle n'est pas d'emblée réalisée, mais elle fait plutôt l'objet d'un devoir (Sollen), d'une tâche infinie assignée au moi pratique. À cet égard, l'organisme incarne de manière analogique la réalisation du but qui doit être celui de la morale, mais qui demeure inaccessible pour elle.

Ainsi, de même que la raison pratique est obligée d'aplanir le conflit entre les lois de la liberté et celles de la nature en les réunissant dans un principe supérieur dans lequel la liberté est elle-même nature et la nature liberté, de même il faut que la raison théorique, dans son usage téléologique, parvienne à un principe supérieur, dans lequel mécanisme et téléologie coïncident, principe qui précisément ne saurait en aucune façon être déterminable en tant qu'objet ${ }^{61}$.

Il va de soi que ce principe supérieur ne peut servir au sujet connaissant de principe déterminant en regard de l'objet. La nature et la liberté, le mécanisme et la finalité ne peuvent être unis que dans ce que Kant appelle un « intellect intuitif», fait d'un être inconditionné, absolu. C'est dire que dans le cas tant de la liberté pratique que de la finalité naturelle, le principe comporte une référence à l'absolu. Les Lettres philosophiques sur le dogmatisme et le criticisme ne définiront-elles pas du reste l'absolu comme l'union de la liberté et de la nécessité ${ }^{62}$ ?

Il convient toutefois d'insister sur une différence notoire entre les deux termes de l'analogie mise en place par Schelling dans $D u$ Moi et c'est la suite du texte qui nous en informe : « Ce qui pour le Moi absolu est accord absolu est pour le Moi fini accord

\footnotetext{
61 Schelling, Vom Ich , SW I/1, 241-242; trad. fr., p. 147, souligné par nous.

62 Schelling, Philosophische Briefe über Dogmatismus und Kriticismus, SW I/1, 330; trad. fr. Lettres philosophiques sur le dogmatisme et le criticisme, dans Premiers écrits (1794-1795), p. 203.
} 
produit, et le principe de l'union qui pour le premier est principe constitutif d'une unité immanente, n'est pour le second que principe régulateur d'une unité objective qui doit devenir immanente ${ }^{63} »$. Pour le Moi fini, l'harmonisation de la loi de la liberté avec celle de la nature est un devoir (Sollen), une quête continuelle, en sorte que, à strictement parler, l'absolu est sur cette voie à jamais hors de portée. Or, il en va tout autrement pour l'organisme. Celui-ci n'incarne certes pas en lui-même l'absolu, mais par l'entrée en action simultanée qu'il présente du mécanisme et de la finalité, il n'en a pas moins la valeur d'un symbole de l'absolu. Voilà qui explique, à notre avis, l'attention portée par Schelling aux phénomènes de la nature organique. Distincte de la finalité morale dont la réalisation nécessite une conquête infinie, la finalité naturelle symbolise d'emblée un absolu, dont elle participe par le fait même.

Il importe d'indiquer ici dans quelle mesure l'organisme, indépendamment de toute considération pratique, peut être considéré en lui-même comme l' "expression » (Ausdruck) phénoménale de l'absolu ${ }^{64}$. Et pour ce faire, il faut être prudent, car on pourrait faire valoir que Fichte confère aussi à l'organisme la valeur d'un analogon de l'absolu, pour autant toutefois que cet absolu soit envisagé sous l'angle du moi pratique. Et c'est là toute la différence. La Doctrine de la science nova methodo caractérise ainsi l'organisme comme un «analogon de la liberté » dans le monde sensible puisque l'on y découvre à l'œuvre une productivité, bien qu'elle soit régie par des lois strictement déterminées $^{65}$. Or, lorsque 1 'on considère les choses de plus près, on se rend compte que ce que la métaphore organique traduit pour Fichte, c'est l'action réciproque des êtres dans

\footnotetext{
63 Schelling, Vom Ich, SW I/1, 242; trad. fr., p. 147-148, souligné par Schelling.

${ }^{64}$ L'Erster Entwurf eines Systems der Naturphilosophie (1799) nous apprend en effet que l'objet de la philosophie de la nature est l'inconditionné, non pas au sens où l'inconditionné s'y " manifeste » directement, mais en ceci que les produits naturels finis en sont « pour ainsi dire une expression particulière ». Cf. $S W \mathrm{I} / 3,11-12$. Dans son Bruno oder über das göttliche und natürliche Princip der Dinge (1802), Schelling reviendra sur l'organisme à titre d'illustration (Abbild) de l'absolu. Dans l'organisme, en effet, possibilité et réalité ne sont pas séparées comme dans un enchaînement mécanique, elles sont simultanées. $S W$ I/4, 250; trad. fr. J. Rivelaygue, Bruno ou du principe divin et naturel des choses, Paris, L'Herne, 1987, p. 80 : «Quelque fini que tu puisses poser, et quelque différence que tu places entre possibilité et réalité effective, il en est comme de l'infinie possibilité du corps entier, que chaque partie d'un organisme contient : à l'égard de ce dernier, et sans référence au temps, la réalité effective est aussi immédiatement posée. Et de même qu'à l'inverse, aucune partie organique isolée n'a sa possibilité avant elle ou hors d'elle-même, mais immédiatement avec elle dans les autres parties, de même aussi pour le fini, en tant qu'il est dans l'absolu, la réalité effective n'est point séparée de la possibilité ni non plus celle-ci de celle-là. »

${ }^{65}$ Fichte, Wissenschaftslehre nova methodo, p. 238; trad. fr., p. 329. De même, dans le brouillon d'une lettre à Schelling du 27 décembre 1800, Fichte prétend découvrir dans la nature un «analogon de notre autodetermination ». Briefwechsel, p. 114; trad. fr., p. 105.
} 
le monde rationnel (Vernunftwelt), et non pas directement l'absolu : « en tout individu se trouve quelque chose qui fait qu'il doit déduire des êtres raisonnables hors de lui; de même, le monde sensible se trouve en action réciproque avec lui-même ${ }^{66}$ ». À partir de là, Fichte conclut qu'il y a trois niveaux d'action réciproque : les rapports mutuels des êtres du monde rationnel, l'action réciproque des parties de l'organisme qui fournit une illustration sensible de la communauté des êtres raisonnables, et enfin l'action réciproque entre ces deux niveaux, entre la liberté propre au monde rationnel et la nature organique qui lui sert de support phénoménal. Or, ce qu'il y a de significatif dans ce passage de la Doctrine de la science nova methodo consacré au statut de la nature organisée, c'est que l'organisme perd bientôt son statut d'analogon de la liberté pour redevenir purement et simplement le point de passage de la liberté dans la nature. Le troisième niveau de l'action réciproque réitère donc, du point de vue transcendantal, cette prise en charge du corps propre par le moi pratique :

Le monde rationnel se trouve en action réciproque avec lui-même, comme le monde sensible, et les deux mondes se trouvent tous deux en action réciproque et apparaissent tels. C'est... dans les corps articulés que nature et liberté s'interpénètrent, à travers la liberté de l'individu; la liberté entière agit dans la nature entière ${ }^{67} \ldots$

Nous retrouvons ici l'organisme comme moyen terme entre la liberté et la nature telle qu'elle se présente dans le Système de l'éthique sous la forme de la tendance. La nature organique, comme c'était le cas dans cette œuvre, n'intéresse pas pour elle-même. Considéré sous l'angle de la morale, l'organisme perd bientôt son statut d'analogon de la liberté pour devenir tout bonnement son instrument. La nature organique chez Fichte ne présente pas d'intérêt philosophique propre, elle ne constitue pas une figure autonome. En ménageant au moi pratique un accès à la nature, elle est mise à profit pour cette tâche qui, en définitive, consiste à faire reculer les frontières de la nature au profit de la culture.

Comme les écrits de Schelling considérés dans la présente recherche s’inscrivent dans la veine de l'idéalisme transcendantal, nous sommes bien évidemment susceptibles d'y découvrir des passages où la nature organique sert à illustrer le moi fini. Les Abhandlungen, par exemple, dépeignent l'organisme comme le « trait confus de l'âme »

${ }^{66}$ Fichte, Wissenschaftslehre nova methodo, p. 238; trad. fr., p. 330.

${ }^{67}$ Fichte, Wissenschaftslehre nova methodo, p. 239; trad. fr., p. 330. 
(verschlungener Zug der Seele) et la vie comme l'« analogon de l'être spirituel ». Et ceci sans compter le fameux passage de l'Introduction aux Idées où Schelling déclare que la nature est l'« esprit visible ${ }^{68} »$. Mais d'autres textes, comme l'écrit Sur le Moi et le Système de l'idéalisme transcendantal ${ }^{69}$, que nous abordons maintenant, trahissent des motivations autres, bien qu'elles ne rompent pas encore le cercle de l'idéalisme subjectif. Ainsi par exemple, dans le Système de l'idéalisme transcendantal, Schelling fait-il état de la « magie » qui entoure depuis toujours la nature organique. Il rappelle également le mot de Kant dans la troisième Critique, selon lequel la nature est une « écriture chiffrée » (Chiffreschrift) qu'il s'agit de décrypter ${ }^{70}$. L'idéalisme transcendantal est sans contredit à même de décoder cette écriture et Schelling est le premier à le reconnaître. Mais reste qu'il semble malgré tout fasciné par le mystère que continue à receler le monde organique et la nature en général, même pour qui l'étudie en profondeur. De ce point de vue, il partage avec Kant cet étonnement devant les produits naturels, même si dans son cas l'intérêt pour ces questions de finalité naturelle ne procède pas d'une inquiétude quant à la réalisation du bonheur empirique. En effet, si Kant est à l'affût de « signes » à l'intérieur de la nature, c'est que sa conception du souverain bien l'y incite. Toutefois, comme Schelling, en accord avec Fichte, tourne le dos à une conception du souverain bien qui réclame en guise de récompense un bonheur empirique proportionnel au mérite moral, son intérêt pour la finalité naturelle n'est pas dicté par de telles attentes ${ }^{71}$. Ainsi que nous l'a enseigné $\mathrm{Du} M o i$, la fascination de Schelling pour la nature tient plutôt à son intérêt pour l'absolu et pour son rapport au fini.

Le chapitre du Système de l'idéalisme transcendantal consacré à la téléologie explique clairement pourquoi aux yeux de Schelling l'organisme représente un

\footnotetext{
68 Schelling, Abhandlungen, SW I/1, 386; Ideen..., SW I/2, 56.

${ }^{69}$ Le thème du moyen terme se trouve dans l'Introduction de cet ouvrage. Mais il intervient précisément en vue d'annoncer la fonction médiane que remplit la téléologie dans le système. Schelling, System des transzendentalen Idealismus, SW I/3, 348-349; trad. fr. C. Dubois, Le système de l'idéalisme transcendantal, Louvain, Peters, 1978, p. 14 : « Il est facile de voir que ce problème ne peut être résolu ni dans la pihilosophie théorique ni dans la philosophie pratique, mais dans une philosophie supérieure qui est le moyen terme [Mittelglied] reliant les deux... » p. 15 : «La philosophie des fins de la nature, ou la téléologie, est donc le point de jonction [Vereinigungspunkt] de la philosophie théorique et de la philosophie pratique. "

${ }^{70}$ Schelling, System des transzendentalen Idealismus, $S W$ I/3, 608; trad. fr., p. 243. Voir aussi pour le thème de l' " énigme du monde » les Philosophische Briefe, $S W$ I/1, 310; trad. fr., p. 180, et pour la nature comme « déesse voilée » les Ideen, $S W \mathrm{I} / 2,12$.
} 
phénomène de l'absolu qui se distingue de l'action morale et qui, à sa manière, s'avère plus fidèle à la chose même. En effet, dans le contexte pratique, le Moi fini est en quête continuelle de la réalisation de la liberté dans le monde, étant constamment partagé entre le devoir moral et les contraintes sensibles. En revanche, l'organisme présente d'un seul tenant le phénomène intégral de l'absolu, dans la mesure où la nécessité et la liberté y sont d'emblée en parfaite cohésion.

Dans le produit naturel se trouve encore uni ce qui est scindé dans l'agir libre en vue du phénomène. Chaque plante est entièrement ce qu'elle doit [soll] être, le libre en elle est nécessaire, et le nécessaire libre. L'homme, lui, est un éternel fragment, car ou bien son agir est nécessaire et dans ce cas non libre, ou bien il est libre et dans ce cas il n'est pas nécessaire ni légal. Seule la Nature organique me donne donc le phénomène complet, dans le monde extérieur, de la réunion de la liberté et de la nécessité ${ }^{72}$.

Si Schelling introduit ce passage en affirmant que l'organisme comme fin naturelle est en fait le « phénomène de la liberté en nous », il faut avouer que ce phénomène est en luimême instructif à plus d'un titre. Cette comparaison est significative dans la mesure où, comme toute comparaison, elle présente un élément discordant. Et Schelling signale sans équivoque en quoi consiste le point de divergence : le sujet agissant apparaît en luimême fragmenté (Bruchstück), partagé qu'il est entre le devoir moral et les penchants naturels, alors que l'organisme est en sa phénoménalité d'entrée de jeu ce qu'il « doit » être. L'organisme peut donc être envisagé comme le phénomène de la liberté pratique, à condition de souligner qu'il est carrément dépourvu de cette dimension propre à l'agir moral qu'est l'ajournement infini de sa complète réalisation. C'est dire que la cohésion parfaite que l'on note entre nécessité et liberté dans la nature organique relève d'un registre autre que celui de la philosophie pratique. Schelling prend en effet bien soin de marquer l'indépendance de la téléologie naturelle en insistant sur le fait qu'elle appartient au domaine de la "philosophie théorique », exactement comme il l'avait fait dans l'écrit Du Moi. Les deux termes de l'analogie sont donc indépendants, au point qu'il est également permis de voir dans la mise en œuvre simultanée du mécanisme et de la finalité au sein de l'organisme une figuration autonome, si indirecte soit-elle, de

\footnotetext{
${ }^{71}$ Schelling, Vom Ich, SW I/1, 197 note, 201, 240; trad. fr., p. 101-102, 106, 146. Philosophische Briefe, SW I/1, 322; trad. fr., p. 193-194.

${ }^{72}$ Schelling, System des transzendentalen Idealismus, SW I/3, 608; trad. fr., p. 243.
} 
l'absolu ${ }^{73}$. En somme, l'analogie établie par Schelling dans le chapitre du Système de l'idéalisme transcendantal consacré à la téléologie ne vise nullement à subordonner les fins naturelles à la finalité pratique. La nature organique y est au contraire envisagée comme un objet propre à la philosophie théorique.

À première vue, il faut convenir que cette lecture de l'extrait en question peut sembler un peu forcée. Tel serait effectivement le cas, à notre avis, si Schelling n'avait pris soin d'indiquer dans la préface de cette oeuvre le sens et la portée qu'il entend conférer à son chapitre sur la téléologie, nous mettant en garde contre toute saisie réductrice du problème de la finalité. Il ne faut pas sous-estimer l'aspect critique de cette préface qui commence par préciser la manière dont Schelling conçoit l'idéalisme transcendantal. C'est qu'en fait cette œuvre vise à élargir (erweitern) le champ de cet idéalisme qui, cela est implicite, s'est jusqu'ici cantonné dans un domaine trop restreint. À ses yeux, l'idéalisme transcendantal n'est pas encore à la hauteur de ses prétentions puisqu'il a eu tendance à négliger son statut de doctrine du savoir (Wissen-schaftslehre). La remarque critique s'adresse donc en large partie à l' « inventeur » (Erfinder) de la Wissenschaftslehre lui-même ${ }^{74}$.

Nous en voulons pour preuve le long passage de la préface qui est consacré à l'organisme et qui anticipe le chapitre sur la téléologie que nous venons de commenter. C'est dire du même coup que la tâche d'expliquer la nature ne revient pas uniquement à la Naturphilosophie (discutée dans cette préface), mais qu'elle incombe aussi à sa contrepartie subjective, l'idéalisme transcendantal, car dans les deux cas il importe de procéder à la « construction des objets ». Pour l'idéalisme transcendantal, la tâche de la connaissance consiste à retracer le « mécanisme de l'intuition » de l'objet, en l'espèce de l'organisme. Or, cette tâche est éminemment théorique et ne peut souffrir quelque interférence que ce soit, notamment de la part de la philosophie pratique. La tentation est

\footnotetext{
${ }^{73}$ Schelling n'afffirmait-il pas dans les Lettres philosophiques que « celui qui a réfléchi sur la liberté et la nécessité aura découvert de lui-même que ces principes doivent nécessairement être réunis [vereinigt] dans l'absolu... »SW I/1, 210; trad. fr., p. 203. Comment dès lors interpréter les mots que nous venons de lire dans le Système de l'idéalisme transcendantal "phénomène complet... de la réunion [vereinigt] de la liberté et de la nécessité », sinon comme une « expression » de l'absolu?

Par ailleurs, pour l'importance du moment « théorique » chez Schelling, voir Klaus-Artur Scheier, « Die Bedeutung der Naturphilosophie im deutschen Idealismus », Philosophia naturalis, vol. 23 (1986), p. 396397.

${ }^{74}$ Schelling, System des transzendentalen Idealismus, $S W$ I/3, 331; trad. fr., p. 2.
} 
grande en effet de passer outre au processus de la constitution de la finalité interne de l'être organisé, pour le soumettre à une finalité externe, celle du sujet moral. À la lumière des publications ultérieures de Schelling, il ne fait aucun doute que dans l'extrait qui suit, c'est Fichte qui est pris à partie, notamment pour la déduction du corps propre (Leib) que l'on trouve dans ses écrits sur le droit et sur l'éthique.

...[M]ême si je démontre, par exemple, qu'il est nécessaire pour les besoins de la liberté ou des fins pratiques qu'il y ait de la matière avec telles ou telles déterminations, ou que l'intelligence intuitionne son agir sur le monde extérieur comme médiatisé par un organisme, cette démonstration ne me donne cependant encore aucune réponse à la question de savoir comment et par quel mécanisme l'intelligence intuitionne précisément cela même qui est nécessaire à cet $\operatorname{effet}^{75}$.

D’après Schelling, ces « déductions » et « Preuves » téléologiques ne sont d'aucun secours dans la philosophie transcendantale et il ne faut pas s'étonner de le voir revenir à la charge avec un argument semblable dans le chapitre sur la téléologie naturelle ${ }^{76}$. En effet, la finalité qui caractérise en propre l'organisme est la finalité interne et une approche du corps articulé qui se place sous le signe de la finalité pratique n'est d'aucune utilité sur le strict plan de la connaissance. Or, l'idéalisme transcendantal se définit d'abord et avant tout comme une entreprise de connaissance, si bien que la spéculation doit seule ici entrer en considération, sans égard à la finalité externe, qu'elle ait pour nom Providence ou liberté morale. Schelling fait donc valoir dans sa préface que dans le champ du savoir, la philosophie pratique ne possède « aucune voix » puisqu'elle ne contribue en rien à nous faire comprendre l' « origine » de la nature organique. Aussi, dans l'ensemble du système de l'idéalisme transcendantal, la philosophie pratique n'estelle qu'une instance intermédiaire, et non l'instance suprême, ce rôle étant réservé, du moins dans ce texte, à l'art ${ }^{77}$.

\footnotetext{
75 Schelling, System des transzendentalen Idealismus, $S W$ I/3, 332-333; trad. fr., p. 4. Voir également la lettre de Schelling à Fichte du 3 octobre 1801, Briefwechsel, p. 140; trad. fr., p. 131 : « Je sais suffisamment bien en quelle petite région de la conscience vous devez situer la nature, avec le concept que vous en avez. Elle n'a pour vous absolument aucune signification spéculative, mais seulement une signification téléologique. Mais votre avis devait-il vraiment être que, par ex., la lumière existe seulement afin que les êtres de raison, en parlant l'un avec l'autre, puissent se voir, et l'air afin que, s'entendant l'un l'autre, ils puissent se parler? ». Cf. Über das Verhältnis der Naturphilosophie zur Philosophie überhaupt (1802), SW I/5, 113-114.; Darlegung des wahren Verhältnisses der Naturphilosophie zur verbesserten Fichteschen Lehre (1806), SW, I/7, 17, 110. Bruno, SW I/4, 326-327; trad. fr., p. 177.

${ }^{76}$ Schelling, System des transzendentalen Idealismus, SW I/3, 608, 610; trad. fr., p. 243, 244.
} 
Notre enquête nous a d'abord permis de découvrir une entente profonde entre Fichte et Schelling sur la façon de développer la théorie de la nature organique. Loin d'être un simple produit artisanal, l'organisme est en effet animé par une finalité interne qui le détermine dans toutes ses parties, même les plus infimes. À l'échelle macroscopique, par ailleurs, les deux penseurs conviennent, nous l'avons vu, que l'univers entier constitue un gigantesque organisme. Et ceci n'a pas de quoi surprendre : si l'intelligence se développe elle-même à la manière d'un organisme, il est normal que la nature, laquelle n'est rien d'autre qu'une projection de l'intelligence, en porte la trace et qu'elle puisse en conséquence faire l'objet d'une connaissance systématique. Or, comme les textes de Schelling que nous avons commentés s'inscrivent dans cette optique, qui est celle de l'idéalisme transcendantal, on comprend pourquoi Fichte ne pouvait trouver de motifs sérieux de s'inquiéter des travaux de son jeune collègue, du moins avant 1800. Ainsi, par exemple, il est devenu clair que les passages des Idées portant sur cet objet en apparence irréductible qu'est l'organisme, n'impliquent nullement le renvoi à une chose en soi qui en serait la cause. Au contraire, l'organisme, parce que le «concept» de fin y joue un rôle essentiel, n'est là que pour une intelligence, si bien que tant sa matière que sa forme sont susceptibles de recevoir une interprétation transcendantale (et non transcendante). À ce compte, Schelling peut encore se prévaloir de son sobriquet de « crieur public du moi ».

Cela étant dit, si nous revenons au constat de Heidegger, que devons-nous conclure? Celui-ci signale en effet que la nature n'a pas véritablement de place dans l'œuvre publiée de Fichte. S'agit-il là d'une lacune purement accidentelle, reliée, par exemple, à l'existence mouvementée qu'a menée Fichte à Iéna et qui l'aurait privé du temps et de la sérénité requis pour mener à terme une telle étude? La seconde partie de notre recherche nous incite plutôt à croire qu'il y a derrière cette négligence des motifs philosophiques profonds, qui tiennent au sens conféré par Fichte à la primauté de la philosophie pratique. Nous avons vu jusqu'à quel point le Système de l'éthique asservit le corps humain, et avec lui la nature entière, aux fins du sujet pratique. Dans la perspective de la philosophie morale, le geste est peut-être légitime, mais la sujétion de l'organisme à une finalité externe ne doit surtout pas rendre superflue, au dire de

${ }^{77}$ Schelling, System des transcendentalen Idealismus, $S W$ I/3, 332, 333; trad. fr., p. $3,5$. 
Schelling, l'approche théorique de la nature organique, laquelle recèle son moment de vérité, ne serait-ce que comme symbole de l'absolu, sans égard à l'action morale. Toute la question réside alors dans la conception que de part et d'autre l'on se fait de l'absolu.

Dans l'échange épistolaire qui à partir de 1800 alimente le débat entre Schelling et Fichte, ce dernier fait remarquer à son correspondant qu'il n'a pas pu s'empêcher de sourciller, à la lecture des Lettres philosophiques sur le criticisme et le dogmatisme, devant l'équivalence qui y est affirmée - sur le plan théorique - entre les deux systèmes de pensée en question. Du côté du dogmatisme, on le sait, la liberté est résorbée dans la nécessité, alors que du côté du criticisme, la nécessité est résorbée dans la liberté. Mais ce qui importe pour Schelling dans ce texte, c'est l'équivalence du résultat : un retour à l'absolu, lui-même défini comme l'identité parfaite de la liberté et de la nécessité. Ce qui est caractéristique ici de la vision schellingienne, c'est le maintien, au sein même de l'absolu, de cette dimension de nécessité inspirée de Spinoza. Il y a pour lui dans l'absolu une nécessité qui est constitutive de son essence, au même titre que la liberté. Or ce constat, banal en apparence, permet de cerner certaines divergences significatives chez nos deux auteurs, divergences qui découlent précisément de leur conception respective de l'absolu. Nous nous bornerons à trois exemples.

Dans sa lettre du 3 octobre 1801, Schelling rappelle à Fichte une thèse que ce dernier avait pu lire dans les Lettres philosophiques : l'absolu n'est pas seulement une « pure agilité », comme le veut Fichte, il est aussi, et au même titre, pur repos : l' « absolue activité » est identique au « repos absolu ${ }^{78} »$. La remarque de Schelling cherche ici bien évidemment à stigmatiser la conception 'activiste' de l'absolu qui se dégage des textes fichtéens et qui a pour conséquence que face au sujet agissant la nature apparaît comme le royaume de la nécessité, voire de la « mort». Un second indice nous permet d'ailleurs de confirmer cette insistance de Schelling à maintenir une équivalence stricte entre la passivité et l'activité au sein de l'absolu. Ainsi présente-t-il une conception du souverain bien où sont maintenus en une parfaite cohésion bonheur et moralité ${ }^{79}$. De toute

\footnotetext{
78 Briefwechsel, p. 133; trad. fr., 125. Philosophische Briefe, SW I/1, 325, 327; trad. fr., p. 197, 199.

79 Voir à ce sujet Reinhard Lauth, « Die erste philosophische Auseinandersetzung zwischen Fichte und Schelling 1795-1797 », Zeitschrift für philosophische Forschung, vol. 21 (1967), p. 366-367. À l'appui de la thèse de Lauth, qui fait abstraction des passages où Schelling adhère à la conception fichtéenne de la Seligkeit, on peut mentionner le passage suivant : Vom Ich, SW I/1, 240-241. Cf. Jean-Marie Vaysse, Totalité et subjectivité. Spinoza dans l'idéalisme allemand, Paris, Vrin, 1994, p. 154-155, 168.
} 
évidence, le bonheur est encore associé chez lui à cette dimension de passivité, qui est entièrement bannie chez Fichte. Le bonheur est pour ce dernier la béatitude que procure l'action en elle-même. Mieux : le bonheur, c'est l'agir lui-même, le fait de surmonter l'obstacle. Il n'y a donc pas de dédoublement chez Fichte : l'action morale occupe ici tout le champ du souverain bien. Enfin, un troisième indice quant au rapport qu'entretiennent cette fois moralité et absolu chez Schelling nous est fourni par Reinhold. Dans une lettre de décembre 1795, le prédécesseur de Fichte à l'Université de Iéna confie à ce dernier que le jeune Schelling semble inverser l'ordre de priorité entre le moi pur et la loi morale : «J'ai cru jusqu’à présent que le moi pur... devait être déduit de la loi morale, et non l'inverse. Je persiste ainsi à croire que le vrai sens de la loi morale est mis en danger lorsque l'on déduit celle-ci du moi absolu posé de manière inconditionnée ${ }^{80}$. » Reinhold fait preuve ici d'une remarquable sensibilité face à ce déplacement qui s'opère subrepticement dans $\mathrm{Du}$ Moi. Aussi Fichte, sans toutefois mentionner le nom de Schelling, s'empresse-t-il de replacer les choses dans leur juste perspective, quelques mois après la réception de la lettre de Reinhold, à l'occasion de la publication de la Seconde introduction à la doctrine de la science. La caution ultime de l'intuition intellectuelle se situe bel et bien dans la loi morale, et non l'inverse.

C'est aussi dans les dernières pages de cette Seconde introduction que Fichte nous fait part de sa conception du moi absolu. Il s'agit du passage bien connu où le moi absolu est présenté comme une idée kantienne, c'est-à-dire comme l'objet d'une tâche infinie. À nouveau, l'absolu est abordé exclusivement dans les termes d'une pensée de l'action. Nous disons ici 'exclusivement' car Schelling nous a enseigné dans Du Moi qu'il est possible de mettre en parallèle deux modes de manifestation du principe absolu : d'une part, bien sûr, la lutte incessante entre la liberté et la nature dans l'action morale, de l'autre, l'interpénétration parfaite du mécanisme et de la finalité dans l'organisme. Or, voilà qui peut contribuer à expliquer la prise de distance de Schelling par rapport à Fichte. L'organisme offre en effet une figuration de l'absolu, et ce en marge du schéma

${ }^{80}$ Lettre de Reinhold à Fichte de décembre 1795, dans Fichte, GA III/2, 438-439. Cf. Reinhard Lauth, « Die erste philosophische Auseinandersetzung zwischen Fichte und Schelling 1795-1797 », p. 347. Sans doute, Reinhold songe-t-il à un passage comme celui-ci : Vom Ich, SW I/1, 198; trad. fr., p. 103 : «Le Moi infini ne connaît donc absolument aucune loi morale et, conformément à sa causalité, il est déterminé uniquement comme puissance absolue, égale à soi-même ». Philosophische Briefe, SW I/1, 324; trad. fr., 
de l'action morale. C'est sans doute aussi ce qui amène Schelling à entreprendre l'étude minutieuse de la nature, puisqu'elle ménage à sa façon une voie d'accès à l'absolu, ou plus précisément à cette conception olympienne de l'absolu que Schelling place au centre de sa philosophie ${ }^{81}$. Et dès lors que l'organisme fournit par lui-même une illustration pertinente du principe absolu, Schelling n'a plus qu'un pas à faire pour accorder à la nature sa propre valence ontologique.

p. 196. Pour un exemple de la subordination de la morale à l'intuition intellectuelle, voir Abhandlungen, SW I $1,420$.

${ }^{81}$ À nouveau, X. Tilliette a vu juste lorsque, commentant la conclusion de $D u$ Moi, il souligne la distinction faite par Schelling entre une «liberté active », fichtéenne, et une liberté qu'il qualifie d'«extatique » et qui est le fait du «Moi absolu suprasensible». X. Tilliette, Schelling, une philosophie en devenir I, p. 74. 